\title{
REVIEW
}

\section{Gene transfer approaches in cancer immunotherapy}

\author{
SS Larin, GP Georgiev and SL Kiselev \\ Institute of Gene Biology Russian Academy of Sciences, Moscow, Russia
}

The idea of enhancing or establishing effective immune response against endogenously developed tumor cells is not novel. More than a hundred years ago, bacterial components were used to develop antitumor immune response. Later, when a number of immune system-effecting cytokines had been discovered, they were used for systemic treatment of cancer patients. However, systemic treatment often resulted in even negative outcome. Recent developments of genetic approaches of cell modifications allowed developing of modern techniques of targeted tumor cell elimination. In the present paper, we review modern trends of the antitumor response enhancement based on immunoregulatory gene transfer into different cell types both in vivo and in vitro. Almost all these approaches are based on the activation of the adaptive arm of the immune system in response to tumor cells. However, recent studies indicate that the innate arm of the immune system, as well as adaptive arm, is involved in tumor suppression. The innate immune system uses nonrearranging germline receptors, which could trigger cellular effector responses that are conditional (or instructive) to the subsequent adaptive immune response. Last years' viewpoints on 'self' and 'non-self' recognition and primary induction of the immune response have changed. The key role of lymphocytes is pathogen recognition and, following immune response induction, switched on the central role of dendritic cells in 'non-self' recognition and induction of both innate and adaptive responses. Moreover, innate response is supposed to be an essential starting point in induction of successful and effective acquired response. Most cancer vaccines do not have 'non-self' marks presentation due to their endogenous origin, thus lacking their effectiveness in the induction of the specific long-lasting immune response. Taking this point into consideration, we can conclude that to make cancer vaccine more effective we have to present tumor antigens, together with the molecules that can potentially activate downstream 'non-self' recognition events not in parallel, but as a consequence of tumor antigen processing and presentation.

Gene Therapy (2004) 11, S18-S25. doi:10.1038/sj.gt.3302365

Keywords: oncoimmunology; innate immunity; danger signal; cytokines; cancer vaccines

\section{Modern view on antitumor immune response}

The last two decades were characterized by intensive studies in the fields of molecular and cellular immunology. A lot of new findings allowed scientists to take a look deeper into the Immune Surveillance Hypothesis. Today, the main role in immunological recognition phenomena, distinguishing 'self' from 'non-self' and activation of immune response, has been drown aside from the key role of lymphocyte to the main role of dendritic cells (DCs) - professional antigen-presenting cells. ${ }^{1,2}$ It was shown that DCs could activate both arms of the immune system: innate $^{3}$ and adaptive, ${ }^{4}$ via recognition of 'non-self' in the presence of the so-called 'danger signals' during DC maturation. Moreover, the evolutionary conservative innate immune response plays an important role in the induction of subsequent acquired immunity and successful memory formation. ${ }^{4,5}$

Knowledge of the molecular mechanisms of immune response allowed investigators to develop a broad list of techniques based on the finetuning of the immune system to treat some not previously curable diseases. One of the promising fields is the control of malignant growth. The idea came out in the early 1900 when for the first time Paul Ehrlich proposed that tumor cells can arise

Correspondence: Dr SS Larin, Institute of Gene Biology RAS, 34/5 Vavilova Street, 119334, Moscow, Russia from the body normal cells, but usually neoplastic cells should be eliminated by the immune system. Later, Lewis Thomas has demonstrated that the main cells involved in tumor elimination are cytotoxic $\mathrm{T}$ lymphocytes (CTLs). Today's knowledge about the broad spectrum of tumor antigens, recognized by the immune system $^{6,7}$ and main principles of immune surveillance, are actively used in development of antitumor immunotherapy. ${ }^{8}$ However, a lot of mismatches and contradictions were found in Ehrlich's hypothesis. The main problem is that the result of interaction between the immune system and new antigen at the periphery depends on the microenvironment of the meeting. ${ }^{9,10}$ Generally, when a pathogen injury (penetrate, invade) the body at the periphery, a lot of 'alarms' or 'danger signals' like reactive oxygen intermediates, extracellularmatrix components, heat-shock proteins, nucleotides, soluble mediators, and cytokines are released in inflammation site. These molecules induce activation and maturation of DCs, whereas antigens derived from pathogens are effectively presented to lymphocytes and the typical result is activation of the corresponding clones of effector cells. ${ }^{11}$ In case of tumor formation, endogenous tumor cell growth occurs without any inflammation and tissue damage, at least in the initial stages. Accordingly, in the absence of 'danger signals', tumor antigens cannot be effectively presented by immature DCs and the typical outcome of this immune 
system 'blindness' is tumor cell recognition as a 'self' and not dangerous. ${ }^{12,13}$

Now, taking together all aspects of oncoimmunology, we can suppose that successful anticancer vaccination is possible, but it is difficult to find the combination of immunostimulatory signals, which would work well and overcome this 'blindness'. At the present moment, thousands of experimental models and hundreds of clinical trials aimed to induce antitumor immunity have been reported. The gross part of such investigations actively involved gene transfer approaches to activate antitumor response. These approaches can be classified in different ways.

In the present review, we attempted to classify them on the basis of the target for gene transfer to achieve immune system activation:

- immunization with genetically modified tumor cells;

- use of autologous or allogeneic genetically modified normal cells (eg fibroblast) for local secretion of immunostimulatory molecules;

- gene modification for antigen processing and presentation by DCs;

- application of gene-targeted effector cells like lymphocytes or natural killer cells (NKs);

- transfer of genes encoding antibodies to known tumor antigens in vivo;

- vaccination with DNA-encoding cancer antigens.

Over the last decade of the 20th century, the most popular vaccine strategy is based on the immunization with the genetically modified tumor cells and dates back to experiments of William Coley in the 1890s. At that time, he tried to activate the immune system with bacterial components, and supposed that immune response at least partially can be transferred to tumor cell rejection. Later, this approach was significantly improved by Lindenmann and Klein. ${ }^{14}$ They have demonstrated that immunization with virus-infected tumor cells representing the complex of noncharacterized tumor antigens on these cells can protect against noninfected parental cells. During such immunization, viral proteins attracted immunocompetent cells in the site of vaccination. T lymphocytes and DC recognized viral antigens in complex with tumor antigens became activated and rejected virus-infected tumor cells, followed by rejection of parental tumor cells. The important resulting component of such approach was cytokine production by activated antigen-presenting cells (APCs) and $\mathrm{T}$ helper cells. At a later time, the idea to mark tumor cells with 'danger flag' developed to compel tumor cells to produce immunostimulatory cytokines by themselves without the use of virus activation of APCs. This fruitful idea gave rise to the studies where tumor cells were modified not with the virus but with the genes encoding such cytokines as interleukin (IL)-2, ${ }^{15-17}$ IL-4, ${ }^{17-19}$ IL$7,{ }^{17,20} \mathrm{IL}-12,{ }^{21}$ granulocyte colony-stimulating factor (G$\mathrm{CSF}){ }^{22}$ granulocyte macrophage colony-stimulating factor (GM-CSF), ${ }^{23}$ interferon (IFN)- $\gamma^{24}$ and a wide list of other genes. It was not absolutely the best choice because an important step of innate immunity activation was missed and immunological mechanisms were directed straight up to CTL and DC activation to develop adaptive immune response, although it was very actively used during the decades. Almost all listed cytokines were successfully tested in phase I-II of gene therapy trials in Europe and USA. ${ }^{25}$

\section{Use of autologous or allogeneic genetically modified normal cells for local secretion of immunostimulatory molecules}

Bubeneik and co-authors proposed the idea to deliver biologically active cytokine at a tumor site without manipulating with tumor cells ex vivo. For this purpose, allogeneic or autologous fibroblasts genetically modified to secrete cytokines were used for injections at the site of tumor. These transfected cells functioned like a source of immunoactivating molecules. Peritumoral administration of retroviral transduced, IL-2-producing fibroblast cells into mice carrying subcutaneous transplants of carcinoma cells inhibited the growth of the tumor. ${ }^{26}$ Later, this approach was successfully exploited in a lot of labs; for example, M Lotze and collaborators demonstrated similar results with the gene encoding IL-12. They also demonstrated that autologous genetically modified fibroblast injections were safe and nontoxic. No lymphoid hyperplasia or tissue necrosis was found in the liver, spleen, or lungs of mice receiving repeated inoculation of IL-12-engineered fibroblasts. Tests of liver and renal function were within the normal range. At the same time, long-term protective antitumor immunity was observed. ${ }^{27}$

\section{Gene modification for antigen processing and presentation by $D C s$}

This was firstly discovered by Steinman. ${ }^{28}$ DCs have become known as professional antigen-presenting cells and inducers of immune response. Moreover, at present, they are recognized as the most important cells in induction of innate and primary adaptive immune responses. ${ }^{1-5}$ Scientists were extremely excited by the possibilities of influencing these cells to enhance tumor antigen presentation. ${ }^{29}$ There is a wide range of studies, which involved DCs targeting tumor antigens. We will focus only on those that involved gene transfer approaches. Pioneered work in this field belongs to the E Gilboa lab. Firstly, the phenomenon was shown for DCs loaded with RNA isolated from tumor cells. In stimulation of CTL response, DCs pulsed with unfractionated RNA (total or polyA+) from OVA-expressing tumor cells were as effective as DCs pulsed with OVA peptide. In the poorly immunogenic, highly metastatic B16/F10.9 mouse melanoma tumor model, a dramatic reduction of lung metastases was observed in mice vaccinated with DCs pulsed with tumor-derived RNA. It is worth mentioning that only total or polyadenylated RNA induced protective vaccination, whereas polyA $(-)$ RNA fraction did not. ${ }^{30}$ Later, the potential opportunity to activate immune response with mRNA-transfected DCs was demonstrated for a number of characterized tumor antigens, for example prostate-specific antigen (PSA), ${ }^{31}$ carcinoembryonic antigen (CEA), ${ }^{32}$ E6 and E7 proteins of human papillomavirus. ${ }^{33} \mathrm{~A}$ clinical trial utilizing such approach also has been reported. ${ }^{32}$ Next modification of the approach was the use of DNAtransfected DCs. For modification, genes encoding cytokines, tumor antigens, and molecules involved in antigen presentation were used..$^{34}$ 


\section{Application of gene-targeted effector cells like} lymphocytes or NKs

Using effector cells directly targeted to tumors came from the fundamental papers of Rosenberg. In his brilliant work, Rosenberg et al for the first time have demonstrated that tumor-infiltrating $\mathrm{T}$ lymphocytes, that carried T-cell receptors specific to tumor antigens, can be cultured to achieve large cell mass ex vivo. Following injection of tumor-specific lymphocytes back to the mice bearing micrometastases has led to significant cure of advanced hepatic metastases and advanced pulmonary metastases. ${ }^{35}$ Today, the genes encoding T- and B-cell receptors to antigens are cloned. Determination of the exact mRNA sequence of receptors to specific cancer antigens gave an opportunity to obtain a large quantity of tumor-targeted CTLs by ex vivo transfection of naive cells, with constructs encoding chimeric receptors to designed antigen. This is a promising approach, but injection of genetically modified effector cells targeted to tumor lacked an important step of tumor recognition by DCs and as a consequence misses memory formation. Nevertheless, some papers reported about successful tumor cell-specific targeting of $\mathrm{T}$ lymphocytes ${ }^{36}$ and inhibition of tumor growth after adoptive transfer of $\mathrm{T}$ hybridoma cells expressing chimeric $\mathrm{scFv} /$ zeta gene composed of the variable regions of an HER-2/neuspecific monoclonal antibody joined to the TCR-zeta chain. ${ }^{37}$

With regard to genetically modified NK cells, it looks like more preferable because NK-mediated tumor rejection is a part of innate antitumor response. Participation of NK cells in tumor lyses should induce local cytokine and chemokine secretion with the subsequent attraction of DCs, resulting in developing of memory cells and strong adaptive response. Development of a fast and simple technique of NK nucleofection ${ }^{38}$ clears the way to the adaptation of the approach based on utilization of human NK cells modified with a chimeric immunoglobulin T-cell receptor. ${ }^{39}$ After resolving the problem with the efficient NK cells genetic modification, this idea will be really attractive.

\section{In vivo transfer of genes encoding antibodies to known tumor antigens}

After the development of the technology of monoclonal antibody humanization, ${ }^{40}$ investigators obtained the fine tool for antigen targeting. Genetically engineered antibody molecules composed of a constant part of human antibody molecule and variable domain of mouse monoclonal antibody against the antigen of interest have generally a long half-life in the human body due to the absence of neutralizing immune response and at the same time maintaining all biological functions of natural antibodies. ${ }^{41}$ A high level of such therapeutic protein can be achieved after in vivo electroporation of plasmid DNA encoding humanized antibody. ${ }^{42,43}$ There are different possible immunological mechanisms of tumor rejection following antibodies encoding gene transfer. First, the mechanism can be based on direct binding of recombinant antibodies with receptors on the surface of tumor cells and subsequent growth inhibition through the growth factor receptor blocking. The best example is Herceptin. Direct binding of this humanized monoclonal antibody to Her-2-positive tumor cells leads to signifi- cant tumor growth inhibition. Second, tumor rejection strategy is based on the development of antibodydependent cellular cytotoxicity. In this case, binding of NK cells Fc surface receptor with the opsonized tumor cells leads to the activation of killing mechanisms directly.

\section{Vaccination with DNA encoding cancer antigens}

The last 15 years were marked by rapid development of a new gene therapy approach termed DNA vaccination. It was started from studies of the early $90 \mathrm{~s},{ }^{44,45}$ and after a short time became a quickly developing field of gene transfer-mediated immunostimulation with special emphasis on infections and malignances. The main advantages of DNA vaccination are relative safety (absence of attenuated or killed pathogens), low cost (missing step of protein preparation), and long storage without loss of activity. All these advantages are combined with very efficient immune response. The important fact defining the effectiveness of DNA vaccination is the presence of unmethylated CpG motives in bacterial DNA, which are one of the best immunostimulatory 'danger signals', involved in innate response activation. ${ }^{46}$ As a consequence, DNA vaccines effectively induce both MHC I and MHC II pathways allowing induction of $\mathrm{CD}^{+}$and $\mathrm{CD} 8^{+} \mathrm{T}$ lymphocytes, leading to the development of cellular and humoral immunity. ${ }^{47}$

The list of diseases cured by DNA vaccination is very broad.$^{48}$ Our interest is focused on cancer treatment. In this case, the immunostimulatory properties of unmethylated plasmid DNA are combined with the genes encoding well-characterized individual or a combination of tumor antigens. In vivo intramuscular injection, electroporation into muscle or skin, and particle-mediated delivery of plasmid $\mathrm{DNA}^{49}$ could potentially induce both arms of the immune system, leading to the tumor antigen recognition and memory formation.

\section{Immune system mediators in gene therapy of cancer}

Coming back to the key role of DCs and to the necessity of innate response in acquired response activation, ${ }^{50}$ we can divide all approaches to activate immune response against tumor in two not equal groups. Bearing in mind that successful immune response usually begins with activation of the innate arm in the presence of 'danger signals', followed by activation of the adaptive arm and memory formation, our division will be based on the level or on the starting point of immunological response. First, a small group of immunostimulatory gene transfer approaches are based on utilization of molecules involved in innate immunity mechanisms. In Table 1, we summarized such approaches. In this small group, molecules involved in the recognition of pathogen-associated molecular pattern or molecules representing pathogen-associated pattern or expressed in response to infection during the first fast step of the innate immune response were used. At the same time, all the above-mentioned molecules play the role of 'danger signals' simulating bacterial infection, and as a result strong immune response against tumor cells 
was observed. All these approaches have at least the same or greater effectiveness than the use of molecules involved in regulation or activation of adaptive response. The second group is represented by the genes that encode a large variety of molecules starting from cytokines: IL-2, IL-12, IFN- $\gamma$, GM-CSF; molecules of major histocompatibility complex (MHC); co-stimulatory molecules such as B-7.1 and B-7.2; genes encoding T-cell receptor, and a lot of other mediators of adaptive immune response. All these molecules are usually involved in direct activation of lymphocytes, CTL induction, and often miss an important step of innate response, antigen processing, and presentation by activated DCs. The use of these genes to obtain local release of cytokines or to obtain expression of cell surface molecules does not consider 'danger signals' that might play an important role at the initial phase of immune response. Nevertheless, these approaches are effective in cancer treatment and they have potencies for future improvement. There is a large number of trials, in which mediators of acquired immunity were used; the bestknown classical works are cited in the second part of Table 1.

There are also a number of studies involving a combination of immunoregulatory molecules. To our mind, they are unfortunately also based on a combination of cytokines and receptors from the second part of the table; in other words, on mediators of adaptive immunity, ${ }^{64-67}$ excluding rare exceptions, which combine molecules of both arms of immune response. ${ }^{68}$ In most cases involving genetic modification, amplification of immune response induced by a combination of immunoregulatory molecules was usually reported. Explanation of immune response amplification is based on the understanding of the molecular mechanisms of immune response and its regulation. For example, IL-2 has been shown as a T-cell growth factor. IL-2 is a main cytokine that induces T-lymphocyte cell cycle from the G1 to S phase, followed by T-cell proliferation. Thus, it is the most important cytokine in the induction of CTL immune response. INF- $\gamma$ was discovered as a factor inhibiting virus replication. It was shown later that INF- $\gamma$ is rather an immunomodulatory cytokine, then antiviral. It activates macrophages, increases the expression of MHC class I and class II molecules, and increases antigen presentation. Thus, a combination of IL-2 and INF- $\gamma$ must activate both antigen presentation and activation of T-effector cells. This strategy proved to be very efficient. ${ }^{64}$

The next example, GM-CSF, was first described as a factor supporting clonal growth and differentiation of bone marrow progenitor cells. GM-CSF effects hematopoietic progenitor cells, granulocytes, and monocytes. It also stimulates the antibacterial and antitumor activities of neutrophils, eosinophils, and macrophages, stimulating cytokine production (TNF- $\alpha$, IL-1, M-CSF, and others). GM-CSF stimulates maturation of DCs and upregulates the surface expression of co-stimulatory molecules, thus enhancing the antigen presentation efficiency of DCs. IL-12 is a cytokine with a broad spectrum of activities and is produced by activated macrophages, B cells, and DCs. Among other activities, this cytokine stimulates the proliferation and cytotoxic activity of $\mathrm{NK}$ and $\mathrm{T}$ cells. It also induces secretion of a range of cytokines, including INF- $\gamma$, which has various secondary actions. Thus, a combination of these cytokines is a powerful tool, as it was reported. ${ }^{65}$ The main cause of such amplifications of the immune

Table 1 Classification of cancer gene therapy trials by approaches directed to stimulate innate or adaptive immune responses

\begin{tabular}{|c|c|c|}
\hline Therapeutic molecule & Treatment scheme & Refs. \\
\hline \multicolumn{3}{|c|}{ Approaches directed to stimulate innate immunity mechanisms } \\
\hline Adenovirus & $\begin{array}{l}\text { Adenoviral vectors delay tumor growth in mice bearing melanomas, through } \\
\text { activation of NK cells }\end{array}$ & [51] \\
\hline Immunostimulatory CpG DNA & Mesothelioma model, anticancer effects of the lipid:DNA complex & [52] \\
\hline Mannan-binding protein & $\begin{array}{l}\text { Recombinant vaccinia virus carrying human MBP intratumoral or subcutaneous } \\
\text { injection }\end{array}$ & [53] \\
\hline Beta-defensin 2 & $\begin{array}{l}\text { DNA immunizations with fusion constructs elicit humoral, protective, and } \\
\text { therapeutic immunity against two different syngeneic lymphomas }\end{array}$ & [54] \\
\hline Tag7/PGRP protein & Vaccination by ex vivo transfected tumor cells & [55] \\
\hline Macrophage inflammatory protein $3 \alpha$ & Intratumoral adenovirus injection & [56] \\
\hline Mycobacterium tuberculosis Ag38 gene & Retroviral vector transduced melanoma cells & [57] \\
\hline RANTES & $\begin{array}{l}\text { Ex vivo fibrosarcoma was genetically modified to express and produce the human } \\
\text { RANTES chemokine stably }\end{array}$ & [58] \\
\hline \multicolumn{3}{|c|}{ Approaches directed to stimulate acquired immunity mechanisms } \\
\hline IL-2 & Retroviral vectors poorly immunogenic tumor cells & [15-17] \\
\hline IL-4, & Transfecting malignant cells to produce IL- 4 & [17-19] \\
\hline IL-7 & $\begin{array}{l}\text { IL-7 gene transfer in patients with metastatic colon carcinoma, renal cell } \\
\text { carcinoma, melanoma, or with lymphoma }\end{array}$ & [20] \\
\hline IL-12 & Ex vivo transformation of murine sarcoma using a polycistronic retroviral vector & [21] \\
\hline INF- $\gamma$ & Retrovirally transduced tumor cells & {$[24]$} \\
\hline B-7.1 and B-7.2 & Autologous transfected tumor cell lines & [59-61] \\
\hline MHCI, MHCII & Plasmid DNA transfection in human melanoma & {$[59,62]$} \\
\hline G-CSF & $\begin{array}{l}\text { Retroviral vector delivery of the human G-CSF gene into the colon } \\
\text { adenocarcinoma }\end{array}$ & [22] \\
\hline GM-CSF & $\begin{array}{l}\text { Mouse models and clinical trial of autologous irradiated tumor cells generated by } \\
\text { ex vivo transfection }\end{array}$ & {$[23,63]$} \\
\hline
\end{tabular}


system response is the involvement of different and independent mechanisms of adoptive immune response.

\section{Innate immunity pattern recognition molecules in gene therapy of cancer}

Knowledge about the importance of innate immunity mechanisms allowed developing a more promising tool based on activation of innate response against tumor, followed by the development reactions of adoptive response such as CTL clonal proliferation and memory formation. ${ }^{55}$ This strategy is based on the utilization of a member of a novel family of pathogen molecular pattern recognition molecules. The first member of this diverse family was cloned from mouse and designated as tag7. ${ }^{69}$ Later, its insect homologue was cloned from insects and pathogen molecular pattern recognition was demonstrated, $^{70}$ thus leading to another name, PGRP, for peptidoglycan recognition protein. Intensive sequencing of mammalian genomes allowed us to identify the mouse tagL gene by EST database analysis. ${ }^{71}$ It was designated tagL because it encodes the protein almost twice larger than Tag7/PGRP-S. Later, a large family of genes was identified in Drosophila and human genomes using the same approach. ${ }^{72,73}$

Mammalian Tag7/PGRP-S has been cloned from mouse, ${ }^{69}$ human, ${ }^{70}$ rat, $^{74}$ and cow. ${ }^{75}$ There are small extracellular proteins (19-24 kDa), which structurally share homology with $\mathrm{T}$ phage lyzozyme but do not possess any amidaze activity. Apart from their ability to recognize oligosaccharides and particularly peptidoglycan $^{70,75}$ cytotoxicity towards mammalian cells, ${ }^{69,75,76}$ bactericidal and bacteriostatic activities ${ }^{75}$ were detected. Tag7/PGRP-S is expressed in the spleen, brain, intestine, ${ }^{69,74,77}$ bone marrow, ${ }^{70}$ in lymphocytes ${ }^{78}$ and neutrophils. $^{70,75}$ Isoforms of mammalian TagL/PGRP-L are rather large proteins, which share strong C-terminal homology with Tag7/PGRP-S but also have transmembrane domain and intracellular $\mathrm{N}$ terminus. ${ }^{71,73}$ TagL/ PGRP-L was found to be both secreted and membranebound proteins recognizing a wide variety of pathogens independently of the surface peptidoglycan. ${ }^{79}$ Actually functions other than oligosaccharide binding are not well documented for the mammalian tag7/PGRP family. However, Drosophila studies allowed establishing important mechanisms of PGRP functioning in innate response in insects. One isoform of PGRP-S initiates activation of the prophenoloxidase cascade ${ }^{80}$ not presented in mammals. Another Drosophila isoform PGRP$\mathrm{SA}$ is required for the activation of the Toll receptor ${ }^{81}$ insect orthologue of mammalian TLRs. Interaction of extracellular PGRP-SA with bacteria activates Drosophila Toll via protease cleavage of Spaetzel. Toll activation initiates signaling that results in the activation of two members of the Rel family transcription factors (similar to mammalian $N F-\kappa B$ ), which upon translocation into the nucleus bind $\kappa \mathrm{B}$ sites and initiate insect immune response. Isoforms of the insect PGRP-L participate in the Imd pathway of Drosophila, somewhat similar to the mammalian TNF receptor activation of $N F-\kappa B^{82-84}$ and mediate antimicrobial gene activation upon Grampositive and negative bacteria recognition. ${ }^{82-85}$
None of these cascades was demonstrated for mammalian immune system yet. However, our recent findings indicate that both human and mouse Tag7 / PGRP-S are able to induce lymphocyte chemotaxis both in vitro and in vivo, and also induce DC maturation via CD80, CD86 co-stimulatory molecule expression (unpublished results). All these data taken together allowed us to assume that presenting of tumor antigens in the context of the molecules that potentially activate innate immunity mechanisms, particularly dendritic cell activation after antigen uptake, may have an advantage in antitumor vaccine preparation. The use of genetic modification of tumor cells by the tag7 expressing construct as a vaccine led to the establishment of longlasting antitumor protective immunity. ${ }^{55}$ Moreover, this effect was T-cell dependent and worked on a different tumor model. At the same time, we did not observe any signs of toxicity. Tumors shrunk due to apoptotic events without the neighboring tissue burden. In prophylactic scheme, more than $60 \%$ of animals remained free of tumors although the use of the GM-CSF gene in the same experiments allowed to keep only $40 \%$ of animals tumor free. Preclinical data encouraged initiating Phase I/II clinical trial of the autologous tag7-modified antitumor vaccine. $^{87}$

\section{Future perspectives of anticancer vaccine development}

The idea to enhance or establish effective immune response against endogenously developed tumor cells is not novel. More than a hundred years ago, bacterial components were used to develop antitumor immune response. Later, when a number of immune systemeffecting cytokines had been discovered, they were used for systemic treatment of cancer patients. Recent developments of genetic approaches of cell modifications allowed developing of modern techniques for targeted tumor cell elimination. Nevertheless, the open question at the moment is the search for the most suitable molecules for activation of the immune system against cancer cells. Unfortunately, most cancer vaccines do not have 'non-self' marks presentation due to their endogenous origin, thus lacking their effectiveness in the induction of the specific long-lasting immune response. In our opinion, the most promising results may be obtained with genes encoding molecules, which are involved in activation of the evolutionary more conservative innate arm of immunity and which are shown to be a starting point in development of adaptive immune response and memory formation. Also, special interest should be focused on a combination of genes encoding regulatory molecules from both arms of the immune system.

Taking it into consideration, we can conclude that to make cancer vaccine more effective we have to present tumor antigens together with the molecules that can potentially activate downstream non-self recognition events not parallel but as a consequence of tumor antigens processing and presentation. It is hoped that these approaches can be developed into effective, safe, and practical treatments for patients with cancer and may be some other diseases. 


\section{Acknowledgements}

We thank Mauro Mezzina for helpful suggestions and for kind patience during all stages of manuscript preparation and reviewing. Studies by the authors that are reported here have been supported by grants from the Moscow Anticancer Program, Russian Foundation for Basic Research and Frontiers in Genetics.

\section{References}

1 Matzinger P. The danger model: a renewed sense of self. Science 2002; 296: 301-305.

2 Medzhitov R, Janeway Jr CA. Decoding the patterns of self and nonself by the innate immune system. Science 2002; 296: 298-300.

3 Aderem A, Ulevitch R. Toll-like receptors in the induction of the innate immune response. Nature 2000; 406: 782-787.

4 Schnare $\mathrm{M}$ et al. Toll-like receptors control activation of adaptive immune responses. Nat Immunol 2001; 2: 947-950.

5 Akira S, Takeda K, Kaisho T. Toll-like receptors: critical proteins linking innate and acquired immunity. Nat Immunol 2001; 2: 675-680.

6 Van Der Bruggen $P$ et al. Tumor-specific shared antigenic peptides recognized by human T cells. Immunol Rev 2002; 188: 51-64.

7 Scanlan MJ et al. Cancer/testis antigens: an expanding family of targets for cancer immunotherapy. Immunol Rev 2002; 188: 22-32.

8 Emens LA, Jaffee EM. Cancer vaccines: an old idea comes of age. Cancer Biol Ther 2003; 2: S161-S168.

9 Ohashi PS et al. Ablation of 'tolerance' and induction of diabetes by virus infection in viral antigen transgenic mice. Cell 1991; 65: 305-317.

10 Miller JF, Morahan G, Allison J. Extrathymic acquisition of tolerance by T lymphocytes. Cold Spring Harb Symp Quant Biol 1989; 54: 807-813.

11 Gallucci S, Matzinger P. Danger signals: SOS to the immune system. Curr Opin Immunol 2001; 13: 114-119.

12 Pardoll DM. Cancer vaccines. Nat Med 1998; 4: 525-531.

13 Khong HT, Restifo NP. Natural selection of tumor variants in the generation of 'tumor escape' phenotypes. Nat Immunol 2002; 3: 999-1005.

14 Lindenmann J, Klein PA. Viral oncolysis: increased immunogenicity of host cell antigen associated with influenza virus. J Exp Med 1967; 126: 93-108.

15 Fearon ER et al. Interleukin-2 production by tumor cells bypasses $\mathrm{T}$ helper function in the generation of an antitumor response. Cell 1990; 60: 397-403.

16 Gansbacher B et al. Interleukin 2 gene transfer into tumor cells abrogates tumorigenicity and induces protective immunity. J Exp Med 1990; 172: 1217-1224.

17 Hock $\mathrm{H}$ et al. Mechanisms of rejection induced by tumor celltargeted gene transfer of interleukin 2, interleukin 4, interleukin 7, tumor necrosis factor, or interferon gamma. Proc Natl Acad Sci USA 1993; 90: 2774-2778.

18 Tepper RI, Pattengale PK, Leder P. Murine interleukin-4 displays potent anti-tumor activity in vivo. Cell 1989; 57: 503-512.

19 Golumbek PT et al. Treatment of established renal cancer by tumor cells engineered to secrete interleukin-4. Science 1991; 254: 713-716.

20 Schmidt-Wolf IG et al. Interleukin-7 gene transfer in patients with metastatic colon carcinoma, renal cell carcinoma, melanoma, or with lymphoma. Hum Gene Ther 1994; 5: 1161-1168.

21 Tahara $\mathrm{H}$ et al. Effective eradication of established murine tumors with IL-12 gene therapy using a polycistronic retroviral vector. I Immunol 1995; 154: 6466-6474.
22 Colombo MP et al. Granulocyte colony-stimulating factor gene transfer suppresses tumorigenicity of a murine adenocarcinoma in vivo. J Exp Med 1991; 173: 889-897.

23 Dranoff $G$ et al. Vaccination with irradiated tumor cells engineered to secrete murine granulocyte-macrophage colonystimulating factor stimulates potent, specific, and long-lasting anti-tumor immunity. Proc Natl Acad Sci USA 1993; 90: 3539-3543.

24 Restifo NP et al. A nonimmunogenic sarcoma transduced with the cDNA for interferon gamma elicits CD8+ $\mathrm{T}$ cells against the wild-type tumor: correlation with antigen presentation capability. J Exp Med 1992; 175: 1423-1431.

25 Rosenberg SA. Progress in human tumour immunology and immunotherapy. Nature 2001; 411: 380-384.

26 Bubenik J et al. Local administration of cells containing an inserted IL-2 gene and producing IL-2 inhibits growth of human tumours in nu/nu mice. Immunol Lett 1988; 19: 279-282.

27 Zitvogel L et al. Cancer immunotherapy of established tumors with IL-12. Effective delivery by genetically engineered fibroblasts. J Immunol 1995; 55: 1393-1403.

28 Steinman RM, Cohn ZA. Identification of a novel cell type in peripheral lymphoid organs of mice. I. Morphology, quantitation, tissue distribution. J Exp Med 1973; 137: 1142-1162.

29 Timmerman JM, Levy R. Dendritic cell vaccines for cancer immunotherapy. Annu Rev Med 1999; 50: 507-529.

30 Boczkowski D, Nair SK, Snyder D. Dendritic cells pulsed with RNA are potent antigen-presenting cells in vitro and in vivo. J Exp Med 1996; 184: 465-472.

31 Heiser A et al. Human dendritic cells transfected with RNA encoding prostate-specific antigen stimulate prostate-specific CTL responses in vitro. J Immunol 2000; 164: 5508-5514.

32 Morse MA et al. Immunotherapy with autologous, human dendritic cells transfected with carcinoembryonic antigen mRNA. Cancer Invest 2003; 21: 341-349.

33 Thornburg $\mathrm{C}$ et al. Induction of cytotoxic $\mathrm{T}$ lymphocytes with dendritic cells transfected with human papillomavirus E6 and E7 RNA: implications for cervical cancer immunotherapy. Immunother 2000; 23: 412-418.

34 Nishioka $\mathrm{Y}$ et al. Induction of systemic and therapeutic antitumor immunity using intratumoral injection of dendritic cells genetically modified to express interleukin 12. Cancer Res 1999; 59: 4035-4041.

35 Rosenberg SA, Spiess P, Lafreniere R. A new approach to the adoptive immunotherapy of cancer with tumor-infiltrating lymphocytes. Science 1986; 233: 1318-1321.

36 Arakawa F et al. Targeting of T cells to CEA-expressing tumor cells by chimeric immune receptors with a highly specific singlechain anti-CEA activity. Anticancer Res 2002; 22: 4285-4289.

37 Gritzapis AD, Mamalaki A, Kretsovali A. Redirecting mouse $\mathrm{T}$ hybridoma against human breast and ovarian carcinomas: in vivo activity against HER-2/neu expressing cancer cells. $\mathrm{Br} \mathrm{J}$ Cancer 2003; 88: 1292-1300.

38 Trompeter $\mathrm{HI}$ et al. Rapid and highly efficient gene transfer into natural killer cells by nucleofection. J Immunol Methods 2003; 274: 245-256.

39 Schirrmann T, Pecher G. Human natural killer cell line modified with a chimeric immunoglobulin T-cell receptor gene leads to tumor growth inhibition in vivo. Cancer Gene Ther 2002; 9: 390-398.

40 Gussow D, Seemann G. Humanization of monoclonal antibodies. Methods Enzymol 1991; 203: 99-121.

41 Hudson PJ. Recombinant antibodies: a novel approach to cancer diagnosis and therapy. Expert Opin Investig Drugs 2000; 9: 1231-1242.

42 Perez N et al. Regulatable systemic production of monoclonal antibodies by in vivo muscle electroporation. Genet Vaccines Ther 2004; $2: 2$. 
43 Tjelle TE et al. Monoclonal antibodies produced by muscle after plasmid injection and electroporation. Mol Ther 2004; 9: 328-336.

44 Wolff JA et al. Direct gene transfer into mouse muscle in vivo. Science 1990; 247: 1465-1468.

45 Tang DC, DeVit M, Johnston SA. Genetic immunization is a simple method for eliciting an immune response. Nature 1992; 356: 152-154.

46 Krieg AM. CpG motifs in bacterial DNA and their immune effects. Annu Rev Immunol 2002; 20: 709-760.

47 Wang $\mathrm{B}$ et al. Vaccination against pathogenic cells by DNA inoculation. Curr Top Microbiol Immunol 1998; 226: 21-35.

48 Donnelly JJ et al. DNA vaccines. Annu Rev Immunol 1997; 15: 617-648.

49 Irvine KR et al. Cytokine enhancement of DNA immunization leads to effective treatment of established pulmonary metastases. J Immunol 1996; 156: 238-245.

50 Diefenbach A, Raulet $\mathrm{DH}$. The innate immune response to tumors and its role in the induction of T-cell immunity. Immunol Rev 2002; 188: 9-21.

51 Ruzek MC et al. Adenoviral vectors stimulate murine natural killer cell responses and demonstrate antitumor activities in the absence of transgene expression. Mol Ther 2002; 5: 115-124.

52 Rudginsky $S$ et al. Antitumor activity of cationic lipid complexed with immunostimulatory DNA. Mol Ther 2001; 4: 347-355.

$53 \mathrm{Ma} Y$ et al. Antitumor activity of mannan-binding protein in vivo as revealed by a virus expression system: mannan-binding protein dependent cell-mediated cytotoxicity. Proc Natl Acad Sci USA 1999; 96: 371-375.

54 Biragyn A et al. Mediators of innate immunity that target immature, but not mature, dendritic cells induce antitumor immunity when genetically fused with nonimmunogenic tumor antigens. J Immunol 2001; 167: 6644-6653.

55 Larin SS et al. Immunotherapy with autologous tumor cells engineered to secrete Tag7/PGRP, an innate immunity recognition molecule. J Gene Med 2004; 6: 798-808.

56 Fushimi $\mathrm{T}$ et al. Macrophage inflammatory protein 3alpha transgene attracts dendritic cells to established murine tumors and suppresses tumor growth. J Clin Invest 2000; 105: 1383-1393.

57 Sfondrini L et al. Anti-tumor immunity induced by murine melanoma cells transduced with the Mycobacterium tuberculosis gene encoding the 38-kDa antigen. Gene Therapy 1998; 5: 247-252.

58 Mule JJ et al. RANTES secretion by gene-modified tumor cells results in loss of tumorigenicity in vivo: role of immune cell subpopulations. Hum Gene Ther 1996; 7: 1545-1553.

59 Pulaski BA, Ostrand-Rosenberg S. Reduction of established spontaneous mammary carcinoma metastases following immunotherapy with major histocompatibility complex class II and B7.1 cell-based tumor vaccines. Cancer Res 1998; 58: 1486-1493.

60 Fenton RT et al. A phase I trial of B7-transfected or parental lethally irradiated allogeneic melanoma cell lines to induce cellmediated immunity against tumor-associated antigen presented by HLA-A2 or HLA-A1 in patients with stage IV melanoma. NCI protocol T93-0161. BRMP protocol 9401. Hum Gene Ther 1995; 6: 87-106.

61 Zitvogel L et al. Interleukin-12 and B7.1 co-stimulation cooperate in the induction of effective antitumor immunity and therapy of established tumors. Eur J Immunol 1996; 26: 1335-1341.

62 Nabel GJ et al. Immune response in human melanoma after transfer of an allogeneic class I major histocompatibility complex gene with DNA-liposome complexes. Proc Natl Acad Sci USA 1996; 93: 15388-15393.

63 Simons JW et al. Bioactivity of autologous irradiated renal cell carcinoma vaccines generated by ex vivo granulocytemacrophage colony-stimulating factor gene transfer. Cancer Res 1997; 57: 1537-1546.
64 Kircheis $\mathrm{R}$ et al. Cytokine gene-modified tumor cells for prophylactic and therapeutic vaccination: IL-2, IFN-gamma, or combination IL-2+IFN-gamma. Cytokines Cell Mol Ther 1998; 4: 95-103.

65 Chong $\mathrm{H}$ et al. Tumour cell expression of B7 costimulatory molecules and interleukin-12 or granulocyte-macrophage colony-stimulating factor induces a local antitumour response and may generate systemic protective immunity. Gene Therapy 1998; 5: 223-232.

66 Parney IF et al. Granulocyte-macrophage colony-stimulating factor and B7-2 combination immunogene therapy in an allogeneic Hu-PBL-SCID/beige mouse-human glioblastoma multiforme model. Hum Gene Ther 1997; 8: 1073-1085.

67 Zitvogel L et al. Interleukin-12 and B7.1 co-stimulation cooperate in the induction of effective antitumor immunity and therapy of established tumors. Eur J Immunol 1996; 26: 1335-1341.

68 Pulaski BA et al. Cooperativity of Staphylococcal aureus enterotoxin B superantigen, major histocompatibility complex class II, and CD80 for immunotherapy of advanced spontaneous metastases in a clinically relevant postoperative mouse breast cancer model. Cancer Res 2000; 60: 2710-2715.

69 Kiselev SL et al. Molecular cloning and characterization of the mouse tag7 gene encoding a novel cytokine. J Biol Chem 1998; 273: 18633-18639.

70 Kang $\mathrm{D}$ et al. A peptidoglycan recognition protein in innate immunity conserved from insects to humans. Proc Natl Acad Sci USA 1998; 95: 10078-10082.

71 Kibardin AV et al. Molecular cloning of a new mouse gene tagL containing a lysozyme-like domain. Dokl Biochem 2000; 372: 103-105.

72 Werner Tet al. A family of peptidoglycan recognition proteins in the fruit fly Drosophila melanogaster. Proc Natl Acad Sci USA 2000; 97: 13772-13777.

73 Liu C et al. Peptidoglycan recognition proteins: a novel family of four human innate immunity pattern recognition molecules. J Biol Chem 2001; 276: 34686-34694.

74 Rehman A et al. The cloning of a rat peptidoglycan recognition protein (PGRP) and its induction in brain by sleep deprivation. Cytokine 2001; 13: 8-17.

75 Tydell CC et al. Isolation, characterization, and antimicrobial properties of bovine oligosaccharide binding protein: a microbicidal granule protein of eosinophils and neutrophils. J Biol Chem 2002; 277: 19658-19664.

76 Sashchenko LP et al. Peptidoglycan recognition protein tag7 forms a cytotoxic complex with heat shock protein 70 in solution and in lymphocytes. J Biol Chem 2004; 279: 2117-2124.

77 Lo $\mathrm{D}$ et al. Peptidoglycan recognition protein expression in mouse Peyer's Patch follicle associated epithelium suggests functional specialization. Cell Immunol 2003; 224: 8-16.

78 Mirkina II et al. Cloning and study of novel mammalian genes with structural homology with phage lysozyme. Genetika 2000; 36: $1492-1500$.

79 Kibardin AV et al. The differentially spliced mouse tagL gene, homolog of tag7/PGRP gene family in mammals and Drosophila, can recognize Gram-positive and Gram-negative bacterial cell wall independently of T phage lysozyme homology domain. J Mol Biol 2003; 326: 467-474.

80 Yoshida H, Kinoshita K, Ashida M. Purification of a peptidoglycan recognition protein from hemolymph of the silkworm, Bombyx mori. J Biol Chem 1996; 271: 13854-13860.

81 Michel $\mathrm{T}$ et al. Drosophila Toll is activated by Gram-positive bacteria through a circulating peptidoglycan recognition protein. Nature 2001; 414: 756-759.

82 Gottar $\mathrm{M}$ et al. The Drosophila immune response against Gramnegative bacteria is mediated by a peptidoglycan recognition protein. Nature 2002; 416: 640-644.

83 Werner $\mathrm{T}$ et al. Functional diversity of the Drosophila PGRP-LC gene cluster in the response to lipopolysaccharide and peptidoglycan. J Biol Chem 2003; 278: 26319-26322. 
84 Choe KM et al. Requirement for a peptidoglycan recognition protein (PGRP) in Relish activation and antibacterial immune responses in Drosophila. Science 2002; 296: 359-362.

85 Ramet $\mathrm{M}$ et al. Functional genomic analysis of phagocytosis and identification of a Drosophila receptor for E. coli. Nature 2002; 416: $644-648$.
86 Moiseynko VM et al. Phase I/II trial of gene therapy with the autologous tumor cells modified with the tag7/PGRP-S gene in patients with the disseminated solid tumors. Ann Oncol 2004, (in press). 